\title{
Local Distortion of M-Conformal Mappings
}

\author{
J. Morais - C.A. Nolder
}

Received: date / Accepted: date

\begin{abstract}
A conformal mapping in a plane domain locally maps circles to circles. More generally, quasiconformal mappings locally map circles to ellipses of bounded distortion. In this work, we study the corresponding situation for solutions to Stein-Weiss systems in the $(n+1) \mathrm{D}$ Euclidean space. This class of solutions coincides with the subset of monogenic quasiconformal mappings with nonvanishing hypercomplex derivatives (named M-conformal mappings). In the theoretical part of this work, we prove that an M-conformal mapping locally maps the unit sphere onto explicitly characterized ellipsoids and vice versa. Together with the geometric interpretation of the hypercomplex derivative, dilatations and distortions of these mappings are estimated. This includes a description of the interplay between the Jacobian determinant and the (hypercomplex) derivative of a monogenic function. Also, we look at this in the context of functions valued in non-Euclidean Clifford algebras, in particular the split complex numbers. Then we discuss quasiconformal radial mappings and their relations with the Cauchy kernel and $p$-monogenic mappings. This is followed by the consideration of quadratic M-conformal mappings. In the applications part of this work, we provide the reader with some numerical examples that demonstrate the effectiveness of our approach.
\end{abstract}

Keywords Clifford analysis · Stein-Weiss systems - monogenic functions · quasiconformal mappings.

J. Morais

Center for Research and Development in Mathematics and Applications (CIDMA)

University of Aveiro, 3810-193 Aveiro, Portugal.

Tel.: +351-234372543

Fax: +351-234370066

E-mail: joao.pedro.morais@ua.pt

C.A. Nolder

Department of Mathematics Florida State University Tallahassee, USA.

E-mail: craiganolder@hotmail.com 


\section{Introduction}

In the literature, quasiconformal mappings have applications in differential geometry, mathematical physics, discrete group theory, and engineering. Quasiconformal mappings are an old subject, but due to their fundamental position within mathematics they continue to play an important role for instance in computer vision and graphics, surface classification, conformal field theory as part of string theory, probability theory, and medical image analysis. In general, the study of quasiconformal mappings is important for the construction of analytic mappings with specified dynamics, mainly because they can be used as coordinate system transformations in the treatment of partial differential equations, see [4]. This applies in particular to 2D quasiconformal geometry, where the powerful methods of complex analysis prove to be very helpful [27]. More recently, further progress has been made towards approximating the recovery of boundary shapes of domains in which inverse problems are defined, e.g. in scattering, diffraction problems and tomography [20-22]. These applications have stimulated a surge of new techniques and have reawakened interest among researchers in the past few years. A breakthrough in the theory of quasiconformal mappings in the plane was made in the early 1930s by H. Grötzsch [12], M.A. Lavrent'ev [25], L.V. Ahlfors [1], and O. Teichmüller [47]. Higher dimensional quasiconformal mappings were first introduced by M.A. Lavrent'ev in 1938 [26], followed over a period of several years by a series of famous works by L.V. Ahlfors [2], F.W. Gehring [10], and J. Väisälä [48, 49]. For a more extensive and detailed treatment of the subject, we refer the interested reader to [42].

Clifford or Dirac analysis is the study of Dirac operators and the properties of the hypercomplex functions in the their kernels, so called monogenic functions. Dirac operators studied here are generalizations of the CauchyRiemann operators in the plane. Hence Dirac analysis is a generalization of complex analysis and many phenomena in the plane have space extensions, see [11]. The analytical theory of monogenic functions is an active area which began some forty years ago. However, it is only recently that there has been renewed interest in extending quasiconformal mappings to 3D (and higher dimensions) within the framework of Clifford (and quaternionic) analyses. Yet a large number of investigations, see $[9,13,24,28,46]$, have been carried out in connection with studying monogenic functions by a corresponding differentiability concept or by the existence of a well defined hypercomplex derivative. All these approaches result in necessary and sufficient conditions for the hypercomplex derivability, generalizing different concepts from complex analysis. Until now, further attempts to characterize monogenic functions via a generalized conformality concept remain unresolved. A first result was shown in the paper [29] based on a generalized aerolar derivative in the sense of Pompeiu. The relation between M-conformal mappings ( $\mathrm{M}$ stands for monogenic) and the geometric interpretation of the hypercomplex derivative complete the theory of monogenic functions by providing an accounting for the still missing geometric characterization of those functions. The main tool in this paper 
is that M-conformal mappings preserve angles where angles must be understood in terms of "Clifford measures". In [7] P. Cerejeiras et al. studied the existence of local homeomorphims for quaternionic Beltrami-type equations, and determined a necessary and sufficient criterion that relates the hypercomplex derivative of a quaternion monogenic function (from $\mathbb{R}^{4}$ to $\mathbb{R}^{4}$ ) and its corresponding Jacobian determinant.

The understanding of monogenic functions as hypercomplex differentiable functions leads to the question of which property generalizes the conformality of complex valued holomorphic functions. It is well known that in spaces $\mathbb{R}^{n+1}$ of dimension $n \geq 2$ the set of conformal mappings is restricted to the set of Möbius transformations and that the Möbius transformations are not monogenic. Hence, one can only expect that monogenic functions represent certain quasiconformal mappings. On the other hand, the class of all quasiconformal mappings is much bigger than the class of monogenic functions. The question arises, do monogenic functions correspond to a special subclass of quasiconformal mappings? The first general results were already shown by H.G. Haefeli who proved in [18] that a monogenic function is related to certain hyperellipsoids. In the special case of M-conformal mappings from $\mathbb{R}^{3}$ to $\mathbb{R}^{3}$, J. Morais et al., see [16], proved that a monogenic function valued in the reduced quaternions (identified with $\mathbb{R}^{3}$ ) with nonvanishing Jacobian determinant locally maps the unit sphere onto explicitly characterized ellipsoids and vice versa; see also [33, Chap. IV] and $[14,15]$. Besides this, methods used in [15] showed that these considerations also include the description of the interplay between the Jacobian determinant and the hypercomplex derivative of a nonsingular monogenic function. Together with the geometric interpretation of the (hypercomplex) derivative, dilatations and distortions of these mappings could be estimated, see [15]. To progress in this direction, in [37] the coefficient of quasiconformality of those mappings was calculated explicitly. This is particularly rewarding since the computation of this coefficient gives us the information of the ratio of the major to minor axes of the aforementioned ellipsoids. An important observation of [6] (cf. [5]) is that monogenic functions can preserve some of the geometrical properties such as length, distance or special angles, while mapping special domains onto the ball. Both papers are mainly concerned with generalizations of the Bergman reproducing kernel approach to numerical mapping problems analogous to the complex Bergman kernel method of constructing the conformal mappings from a domain to the disk; see also [36] for details about the geometric properties of a monogenic Szegö kernel for 3D prolate spheroids. In this line of investigation, in [17] it is proved that solid angles are not invariant under M-conformal mappings. It is shown that the change of the solid angle depends on one real parameter only. In particular, it is proved that a certain change of the solid angle is necessary and sufficient for the property of the function to be monogenic and orthogonal to the (infinite dimensional subspace) of paramonogenic constants (i.e monogenic functions with identically vanishing hypercomplex derivative). First global results were considered by H. Malonek et al. in [30,31]; see also [3, 8]. The authors studied the global behavior of higher dimensional analogues 
of the exponential function and the classical Joukowski transformation in the quaternionic and Clifford analyses contexts.

In continuation of [35], we extend the results of [16] to the corresponding situation in the $(n+1) \mathrm{D}$ underlying Euclidean space. First, in Section 2 we provide the relevant mathematical background of Clifford analysis and prove some results for M-conformal mappings to be used throughout the paper. The components of a monogenic function are harmonic. As such M-conformal mappings are then a subclass of harmonic mappings. Since we are interested in the local distortion of monogenic functions it is natural to study linear monogenic spaces. We show that the Jacobi matrix associated with a nonsingular monogenic function valued in a Clifford algebra is symmetric with identical diagonal elements. The leading local distortion is then expressed by the corresponding eigenvalues. Section 3 reviews 3D M-conformal mappings and some of their properties. This is followed by the consideration of the local properties of monogenic functions valued in a Clifford algebra. In the theoretical part of this work, we prove that a monogenic function from $\mathbb{R}^{n+1}$ to $\mathbb{R}^{n+1}$ with nonvanishing Jacobian determinant locally maps the unit sphere onto explicitly characterized ellipsoids and vice versa (see Theorem 8 and Remark 7 below). The relations of the semiaxes of the ellipsoids are then expressed through the dimension of the underlying space. Together with the geometric interpretation of the hypercomplex derivative, we deduce dilatations and distortions of these mappings. This includes the description of the interplay between the Jacobian determinant and the hypercomplex derivative of an M-conformal mapping (see Theorem 11 below). Also, we look at this in the context of functions valued in non-Euclidean Clifford algebras, in particular in $\mathcal{C} \ell_{1,0}$ (see Subsection 2.1 above) that is a projective space over the split complex numbers. This is a natural space on which to develop a function theory. In Section 4 we discuss quasiconformal radial mappings and their relations with the Cauchy kernel and $p$-monogenic mappings. Then in Section 5 we discuss quadratic M-conformal mappings. Finally, in Sections 6 and 7 we illustrate our approach using examples produced by Maple and show how these techniques extend to analytic functions in non-Euclidean Clifford algebras.

The broader impacts of this study include the introduction of quasiconformal techniques into Clifford analysis. This will be fruitful both to enrich the understanding of the geometrical properties of these analytic space mappings and as tools in such applications of Clifford analysis as image processing and shape analysis. These connections will be revealed to mathematicians, who are working now in what has been largely separate areas, through subsequent publications. The approach we take in deriving the local distortion of M-conformal mappings is more involved that the one in [16], as is not dictated by the dimension of the underlying space. The derivation of those properties is also different than those suggested in [16], and is based on certain classical algebraic manipulations using matrix representations. Of course it is expected that the popularity of M-conformal mappings will grow in the future, due to their promising applications in many areas such as in elliptic partial differential equations and differential geometry. 


\section{Mathematical Preliminaries}

\subsection{Notations and Terminology}

The present subsection collects some definitions and summarizes several algebraic facts of a special Clifford algebra of signature $(0, n)$ to be used in the rest of the paper.

Let $\mathcal{C} \ell_{0, n}$ be the real universal Clifford algebra over $\mathbb{R}$. This Clifford algebra is generated over $\mathbb{R}$ by the standard basis $\left\{e_{0}, e_{1}, e_{2}, \ldots, e_{n}\right\}$ subject to the following relations:

$$
e_{i} e_{j}+e_{j} e_{i}=-2 \delta_{i j} e_{0}, \quad i, j=1, \ldots, n .
$$

We write $e_{0}$ for 1 . The dimension of $\mathcal{C} \ell_{0, n}$ is $2^{n}$. We have an increasing tower $\mathbb{R} \subset \mathbb{C} \subset \mathbb{H} \subset \mathcal{C} \ell_{0,3} \subset \cdots$. Here $\mathbb{H}$ denotes the skew field of Hamiltonian quaternions.

Remark 1 The Clifford algebra $\mathcal{C} \ell_{1,0}$ is spanned by 1 and $j$ where $j^{2}=1$. Therefore $\mathcal{C} \ell_{1,0}$ is isomorphic, as an associative algebra, to the split complex numbers. With the definitions $j^{ \pm}=(1 \pm j) / 2$, the set $\left\{j^{+}, j^{-}\right\}$is a basis of $\mathcal{C} \ell_{1,0}$ with the properties: $\left(j^{+}\right)^{2}=j^{+},\left(j^{-}\right)^{2}=j^{-}, j^{+} j^{-}=j^{-} j^{+}=0$, $j^{+}=j^{-}$, and $j^{+}+j^{-}=1$.

The Clifford algebra $\mathcal{C} \ell_{0, n}$ is a graded algebra as $\mathcal{C} \ell_{0, n}=\oplus_{l} \mathcal{C} \ell_{0, n}^{l}$ where $\mathcal{C} \ell_{0, n}^{l}$ are those elements whose reduced Clifford products have length $l$. We use the conjugation $\overline{\left(e_{j_{1}} \ldots e_{j_{l}}\right)}=(-1)^{l} e_{j_{l}} \ldots e_{j_{1}}$.

For any $A \in \mathcal{C} \ell_{0, n}, \operatorname{Sc}(A)$ denotes the scalar part of $A$, that is the coefficient of the element $e_{0}$. The scalar part of a Clifford inner product, $\operatorname{Sc}(\bar{A} B)$, is the usual inner product in $\mathbb{R}^{2^{n}}$ when $A$ and $B$ are identified as vectors. We will denote this usual inner product as $\langle A, B\rangle$. From now on we will identify $\mathbb{R}^{n+1}$ with the paravector space

$$
\mathcal{A}_{n}:=\operatorname{span}_{\mathbb{R}}\left\{1, e_{1}, e_{2}, \ldots, e_{n}\right\} \equiv \mathcal{C} \ell_{0, n}^{0} \oplus \mathcal{C} \ell_{0, n}^{1} \subset \mathcal{C} \ell_{0, n} .
$$

The elements of $\mathcal{A}_{n}$ are usually called paravectors, and are of the form

$$
x:=x_{0}+x_{1} e_{1}+\cdots+x_{n} e_{n} .
$$

Let $\Omega$ denote an open subset of $\mathbb{R}^{n+1}$ with a piecewise smooth boundary which contains the origin. Throughout the paper, we consider functions $\mathcal{A}_{n}$-valued defined in $\Omega$, i.e. functions of the form

$$
f(x):=u_{0}(x)+\sum_{i=1}^{n} u_{i}(x) e_{i},
$$

where each $u_{l} \in C^{\infty}(\Omega)(l=0, \ldots, n)$ is a function real-valued defined in $\Omega$. The conjugate of $f$ is given by $\bar{f}=u_{0}-\sum_{i=1}^{n} u_{i}(x) e_{i}$, and the norm $|f|$ of $f$ is defined by $|f|^{2}=f \bar{f}=\bar{f} f=\sum_{l=0}^{n} u_{l}^{2}$. Properties (like integrability, continuity or differentiability) that are ascribed to $f$ have to be fulfilled by all components $u_{l}$. 
We further introduce the linear Hilbert space of square integrable functions $\mathcal{A}_{n}$-valued defined in $\Omega$, that we denote by $L^{2}\left(\Omega ; \mathcal{A}_{n}\right)$. Now, let $B^{n+1}$ denote the open unit ball in $\mathbb{R}^{n+1}$. In this assignment, the scalar inner product is defined by

$$
<f, g>_{L^{2}\left(B^{n+1} ; \mathcal{A}_{n}\right)}:=\int_{B^{n+1}} \operatorname{Sc}(\bar{f} g) d V,
$$

where $d V$ denotes the volume of $B^{n+1}$ normalized so that $V\left(B^{n+1}\right)=1$.

We write $M_{(n+1)}(\mathbb{R})$ for the space of $(n+1) \times(n+1)$ real matrices. With matrix addition $M_{(n+1)}(\mathbb{R})$ is a real vector space of dimension $(n+1)^{2}$. We write the $(n+1) \times(n+1)$ reflection matrix as $R_{n}=\operatorname{diag}(-1,1, \ldots, 1)$ throughout. Notice that $\operatorname{det} R_{n}=-1$. Further we use the mapping

$$
\phi: M_{(n+1)}(\mathbb{R}) \longrightarrow M_{(n+1)}(\mathbb{R})
$$

defined as $\phi(A):=R_{n} A$. The mapping $\phi$ is a linear automorphism of $M_{(n+1)}(\mathbb{R})$.

We define the linear space of linear functions taking values in $\mathcal{A}_{n}$, by

$$
\mathcal{L}_{n}:=\left\{f(x)=\left(1, e_{1}, \ldots, e_{n}\right) A x^{T} \mid A \in M_{(n+1)}(\mathbb{R})\right\} .
$$

The map $\psi: M_{(n+1)}(\mathbb{R}) \longrightarrow \mathcal{L}_{n}$ defined as

$$
\psi(A):=\left(1, e_{1}, \ldots, e_{n}\right) A x^{T}
$$

is a linear isomorphism. As such $\operatorname{dim} \mathcal{L}_{n}=(n+1)^{2}$. Via $\psi$, the action of $\phi$ on $M_{(n+1)}(\mathbb{R})$ lifts to $\mathcal{L}_{n}$ as negative Clifford conjugation.

For the sake of completeness, we now recall some more definitions and notations which will be needed through the text. We will make use of the generalized Cauchy-Riemann operator

$$
D_{n}=\frac{\partial}{\partial x_{0}}+\sum_{i=1}^{n} e_{i} \frac{\partial}{\partial x_{i}},
$$

with conjugate

$$
\overline{D_{n}}=\frac{\partial}{\partial x_{0}}-\sum_{i=1}^{n} e_{i} \frac{\partial}{\partial x_{i}} .
$$

The operators $D_{n}$ and $\overline{D_{n}}$ correspond, respectively, to the generalization of the classical Cauchy-Riemann operator $\partial_{\bar{z}}=\frac{1}{2}\left(\frac{\partial}{\partial x}+i \frac{\partial}{\partial y}\right)$ and its conjugate $\partial_{z}=\frac{1}{2}\left(\frac{\partial}{\partial x}-i \frac{\partial}{\partial y}\right), z=x+i y \in \mathbb{C}$.

For a real-differentiable function $f: \Omega \subset \mathbb{R}^{n+1} \rightarrow \mathcal{A}_{n}$ that has continuous first partial derivatives, we define the linear paravector-valued monogenic and anti-monogenic function spaces, respectively, by

$$
\mathcal{M}_{n}:=\left\{f \in \mathcal{L}_{n} \mid D_{n} f=0 \text { in } \Omega\right\},
$$

and

$$
\overline{\mathcal{M}_{n}}:=\left\{f \in \mathcal{L}_{n} \mid \overline{D_{n}} f=0 \text { in } \Omega\right\} .
$$


More generally,

Definition 1 Let $p \neq 1$. A function $f: \Omega \rightarrow \mathcal{A}_{n}$ is $p$-monogenic in $\Omega$ when $D_{n}\left(|f|^{p-2} f\right)=0$ in $\Omega$.

The $p$-Cauchy-Riemann equation has interesting conformal invariance properties and was introduced in [38]. See also [39] and [40] for more general such nonlinear equations.

An important observation shows that the equations

$$
D_{n} f=f D_{n}=0
$$

are equivalent to the system

$$
\text { (R) }\left\{\begin{array}{l}
\sum_{l=0}^{n} \frac{\partial u_{l}}{\partial x_{l}}=0, \\
\frac{\partial u_{m}}{\partial x_{l}}-\frac{\partial u_{l}}{\partial x_{m}}=0 \quad(m \neq l, 0 \leq m, l \leq n),
\end{array}\right.
$$

or, equivalently, in a more compact form:

$$
\left\{\begin{array}{l}
\operatorname{div} \bar{f}=0 \\
\operatorname{rot} \bar{f}=0
\end{array} .\right.
$$

Recall that $\bar{f}$ is said to be a Riesz system of conjugate harmonic functions in the sense of Stein-Weiß $[44,45]$, and system $(\mathrm{R})$ is called the Riesz system [43]. It is a historical precursor that generalizes the classical Cauchy-Riemann system in the plane.

Remark 2 In the case of split complex numbers we define the operators $\bar{\nabla}=$ $\partial_{x}-j \partial_{y}$, with conjugate $\nabla=\partial_{x}+j \partial_{y}$. Notice that they factor the wave equation $\bar{\nabla} \nabla=\partial_{x}^{2}-\partial_{y}^{2}$.

Definition 2 A function $f: \Omega \subset \mathbb{R}^{2} \rightarrow \mathcal{C} \ell_{1,0}$ is split analytic in $\Omega$ when $\bar{\nabla} f=0$ in $\Omega$.

Definition 3 A function $f: \Omega \subset \mathbb{R}^{2} \rightarrow \mathcal{C} \ell_{1,0}$ is hyperharmonic in $\Omega$ if $\left(\partial_{x}^{2}-\right.$ $\left.\partial_{y}^{2}\right) f=0$ in $\Omega$.

Remark 3 It is clear that the components of a split analytic function are hyperharmonic.

A central property in the theory of monogenic functions is the hypercomplex derivability. This question was first studied by A. Sudbery [46] for monogenic functions from $\mathbb{R}^{4}$ to $\mathbb{H}$ and I. Mitelman and M. Shapiro in [32]. In 1999 it was shown in the paper [13] that $\frac{1}{2} \overline{D_{n}} f$ defines the hypercomplex derivative of a monogenic function in all dimensions.

Definition 4 Let $f$ be a monogenic $\mathcal{A}_{n}$-valued function. $\left(\frac{1}{2} \overline{D_{n}}\right) f$ is called hypercomplex derivative of $f$. 
2.2 Influence of the Linear Part of a Monogenic Function

For brevity, assume in the sequel that $f: \Omega \rightarrow \mathcal{A}_{n}$ is such that $f(0)=0$. It is then clear that the invariance of the origin guarantees that the interior of a ball will be mapped to the interior of the image domain. Furthermore, let $J_{f}(x)=\operatorname{det}\left(\partial_{x_{j}} u_{i}\right)_{i, j=0}^{n}$ be the functional determinant ("Jacobian") of $f$. The Jacobian is positive for sense preserving and negative for sense reversing mappings. Hence $f-f(a)$ has the Taylor series near the point $a \in \Omega$

$$
f(x)=T_{1}(x)+\mathcal{R}(x) .
$$

Here $T_{1}$ is the first order Taylor polynomial of $f$ and $\mathcal{R}$ denotes the remaining part. For simplicity of presentation, we assume that $a=0$. We use this simplification because the displacement of center of the image domain will not add anything essential. Notice that $D_{n} \mathcal{R}(0)=0$. Hence

$$
D_{n} f(0)=D_{n} T_{1}(0)
$$

Moreover, $f$ and $T_{1}$ have the same Jacobi matrix and therefore the same Jacobian at $0,\left.J_{f}(x)\right|_{x=0}$, as mappings from $\Omega$ into $\mathbb{R}^{n+1}$. Hence the linearization $T_{1}$ determines the local distortion of the mapping. Since the mappings we consider here are smooth, they are quasiconformal in a domain when they are homeomorphisms with bounded local dilatation throughout the domain. We will calculate these dilatations by computing eigenvalues.

Definition 5 A function $f: \Omega \rightarrow \mathcal{A}_{n}$ realizes an M-conformal mapping if it has a nonvanishing hypercomplex derivative.

Remark 4 Since we have the factorization $\overline{D_{n}} D_{n}=D_{n} \overline{D_{n}}=\triangle_{n}$, the Mconformal mappings are a subclass of the harmonic mappings.

Example 1 The function $f(x)=\left(2 x_{0}, x_{1}, x_{2}\right)$ is an example of an M-conformal mapping in $\mathbb{R}^{3}$ since $2 x_{0}+x_{1} e_{1}+x_{2} e_{2}$ is monogenic. The reflection $g(x)=$ $\left(x_{0}, x_{1}, x_{2},-x_{3}\right)$ is a monogenic mapping in $\mathbb{R}^{4}$.

To proceed further we formulate the following auxiliary results.

Theorem 1 Assume that $f \in \mathcal{L}_{n}$. Then $f \in \mathcal{M}_{n}$ with matrix $A$ if and only if $\tilde{A}=R_{n} A$ is symmetric and traceless. Whereas $f \in \overline{\mathcal{M}_{n}}$ with matrix $B$ if and only if $B$ is symmetric and traceless.

Theorem 2 The mapping $\eta: \mathcal{M}_{n} \rightarrow \overline{\mathcal{M}_{n}}$ given by

$$
\eta(f):=\psi \circ \phi \circ \psi^{-1}(f)=\psi \circ \phi(A)=\psi \circ R_{n} A
$$

is a linear isomorphism.

Remark 5 We have that $\operatorname{dim} \mathcal{M}_{n}=\frac{(n+1)(n+2)}{2}-1=\frac{n(n+3)}{2}$. 
Definition 6 We define the space of paramonogenic mappings, as

$$
\mathcal{H}_{n}:=\mathcal{M}_{n} \bigcap \overline{\mathcal{M}_{n}}
$$

Since $D_{n}+\overline{D_{n}}=2 \frac{\partial}{\partial x_{0}}$, the paramonogenic maps are independent of $x_{0}$. In the plane this space contains only the zero map. The matrix representations of functions in $\mathcal{H}_{n}$ are of the form $A=\operatorname{diag}(0, B)$ where $B$ is symmetric and traceless. For example when $n=2$ they have the form

$$
A=\left(\begin{array}{ccc}
0 & 0 & 0 \\
0 & a & b \\
0 & b & -a
\end{array}\right) \quad(a, b \in \mathbb{R})
$$

We have that $\operatorname{dim} \mathcal{H}_{n}=\frac{(n-1)(n+2)}{2}$.

Theorem 3 Let $f \in \mathcal{M}_{n}$. The following conditions are equivalent:

1. $f \in \mathcal{H}_{n}$;

2. $R_{n} A=A$;

3. $A=\operatorname{diag}(0, B), \quad R_{n-1} B \in \mathcal{M}_{n-1}$.

With $\langle f, g\rangle_{L^{2}\left(B^{n+1} ; \mathcal{A}_{n}\right)}$ defined as $(1), \mathcal{L}_{n}$, and its subspaces $\mathcal{M}_{n}$ and $\mathcal{H}_{n}$, are inner product spaces.

Definition 7 For a subspace $H \subset \mathcal{L}_{n}$ the orthogonal complement is

$$
H^{\perp}:=\left\{f \in \mathcal{L}_{n} \mid\langle f, g\rangle_{L^{2}\left(B^{n+1} ; \mathcal{A}_{n}\right)}=0, \forall g \in H\right\} .
$$

We further define the following linear function subspaces: $\mathcal{Q}_{n}:=\mathcal{H}_{n}^{\perp} \cap \mathcal{M}_{n}$ and $\overline{\mathcal{Q}_{n}}:=\mathcal{H}_{n}^{\perp} \cap \overline{\mathcal{M}_{n}}$. We are thus led to the following result.

Theorem 4 Assume that $f \in \mathcal{L}_{n}$. Then

$$
\mathcal{Q}_{n}=\left\{f \in \mathcal{L}_{n} \mid \psi^{-1}(f)=\operatorname{diag}(n a, a, \ldots, a), a \in \mathbb{R}\right\} .
$$

We end this section by recalling some definitions and notations which will be needed later in the text.

Definition 8 Let $\Omega_{1}$ and $\Omega_{2}$ be open subsets of $\mathbb{R}^{n+1}$ and $f: \Omega_{1} \rightarrow \Omega_{2}$ a diffeomorphism. When $f \in \mathcal{M}_{n}$ or $\overline{\mathcal{M}_{n}}$ with matrix $A$ so that $\operatorname{det} A \neq 0$, we define the inner and outer dilatations of $f$ respectively by

$$
d_{I}(f):=\frac{\left|\lambda_{1} \cdots \lambda_{n+1}\right|}{\left(\min _{j}\left|\lambda_{j}\right|\right)^{n+1}}, \quad d_{O}(f):=\frac{\left(\max _{j}\left|\lambda_{j}\right|\right)^{n+1}}{\left|\lambda_{1} \cdots \lambda_{n+1}\right|}
$$

where the $\lambda_{i}$ 's are the eigenvalues of $R_{n} A$. The maximum dilatation of $f$ is defined by $d(f):=\max \left\{d_{I}(f), d_{O}(f)\right\}$. More generally, if $f$ is monogenic, then we define $d(f)=d\left(T_{1}\right)$.

Definition 9 Let $\Omega_{1}$ and $\Omega_{2}$ be open subsets of $\mathbb{R}^{n+1}$ and $f: \Omega_{1} \rightarrow \Omega_{2}$ a diffeomorphism. The mapping $f \in \mathcal{M}_{n}$ or $\overline{\mathcal{M}_{n}}$ is said to be quasiconformal if $d(f)$ is bounded in $\Omega_{1}$. It is $K$-quasiconformal if $d(f) \leq K<\infty$.

Theorem 5 If $f \in \mathcal{Q}_{n}$ or $\overline{\mathcal{Q}_{n}}$ with $\left.J_{f}(x)\right|_{x=0} \neq 0$, then $d(f)=n^{n}$. 


\section{An Insight into M-Conformal Mappings}

\subsection{The Situation in $\mathbb{R}^{3}$ Revisited}

One of the most interesting points of a holomorphic function is that it realizes in a domain $\Omega \subset \mathbb{R}^{2}$ a conformal mapping providing that its complex derivative exists and is not equal to zero in $\Omega$. This usual treatment includes the description of the connection between the Jacobian determinant and the derivative of such a function.

Suppose that

$$
f: \Omega \subset \mathbb{R}^{2} \rightarrow \mathbb{C} \cong \mathcal{A}_{1}, \quad f:=a_{0} x_{0}+a_{1} x_{1}+\left(b_{0} x_{0}+b_{1} x_{1}\right) e_{1},
$$

with $\left.J_{f}(x)\right|_{x=0} \neq 0$. Here $D_{1}=\frac{\partial}{\partial x_{0}}+e_{1} \frac{\partial}{\partial x_{1}}$. Then $D_{1} f=0$ implies that $f=A x$, where $A=\left(\begin{array}{cc}a_{0} & a_{1} \\ -a_{1} & a_{0}\end{array}\right)$. In this case we have

$$
A=\left(\begin{array}{cc}
-1 & 0 \\
0 & 1
\end{array}\right) \mathcal{O}\left(\begin{array}{cc}
-\sqrt{a_{0}^{2}+a_{1}^{2}} & 0 \\
0 & \sqrt{a_{0}^{2}+a_{1}^{2}}
\end{array}\right) \mathcal{O}^{T},
$$

where $\mathcal{O}$ is the orthogonal matrix of eigenvectors. Hence $A$ is the composition of a dilation with reflections and so maps circles to circles. If instead we have $\overline{D_{1}} f=0$, then $f(x)=\tilde{A} x$, where $\tilde{A}=\left(\begin{array}{cc}-1 & 0 \\ 0 & 1\end{array}\right) A$.

The question that led to [16] was to check whether monogenic mappings from $\mathbb{R}^{3}$ to $\mathbb{R}^{3}$ can also be characterized by some directly visible geometric properties. As shown in [16] there is a great difference in the geometric properties of holomorphic 2D conformal mappings and 3D M-conformal Mappings; see also [33, Chap. IV]).

Take a general linear function $\mathcal{A}_{2}$-valued

$$
\begin{aligned}
f\left(x_{0}, x_{1}, x_{2}\right) & =a_{0} x_{0}+a_{1} x_{1}+a_{2} x_{2}+\left(b_{0} x_{0}+b_{1} x_{1}+b_{2} x_{2}\right) e_{1} \\
& +\left(c_{0} x_{0}+c_{1} x_{1}+c_{2} x_{2}\right) e_{2}
\end{aligned}
$$

with $\left.J_{f}(x)\right|_{x=0} \neq 0$. Here $D_{2}=\frac{\partial}{\partial x_{0}}+e_{1} \frac{\partial}{\partial x_{1}}+e_{2} \frac{\partial}{\partial x_{2}}$. The condition $D_{2} f=0$ implies that

$$
A=\left(\begin{array}{ccc}
\left(b_{1}+c_{2}\right) & a_{1} & a_{2} \\
-a_{1} & b_{1} & b_{2} \\
-a_{2} & b_{2} & c_{2}
\end{array}\right)=\left(\begin{array}{ccc}
-1 & 0 & 0 \\
0 & 1 & 0 \\
0 & 0 & 1
\end{array}\right) \mathcal{O}\left(\begin{array}{ccc}
\lambda_{1} & 0 & 0 \\
0 & \lambda_{2} & 0 \\
0 & 0 & \lambda_{3}
\end{array}\right) \mathcal{O}^{T}
$$

We may assume that $\lambda_{1} \geq \lambda_{2} \geq \lambda_{3}$. Hence $A$ maps the unit sphere onto a specific type of ellipsoids with the property that the length of one of the semiaxes is equal to the sum of the other two.

If instead we have $\overline{D_{2}} f=0$, then $f(x)=\tilde{A} x$, where

$$
\tilde{A}=\left(\begin{array}{ccc}
-1 & 0 & 0 \\
0 & 1 & 0 \\
0 & 0 & 1
\end{array}\right) A
$$


Collecting these ideas, in [16] it is shown that $f: \mathbb{R}^{3} \rightarrow \mathcal{A}_{2}$ realizes locally in the neighborhood of a fixed point $x=x^{*}$ an M-conformal mapping if and only if $\left.J_{f}(x)\right|_{x=x^{*}} \neq 0$. More precisely,

Theorem 6 Let $f$ be a real analytic mapping from $\mathbb{R}^{3}$ to $\mathcal{A}_{2}$ with $\left.J_{f}(x)\right|_{x=0}$ $\neq 0$. Then, $f$ is monogenic if and only if it locally maps small balls onto ellipsoids with the property that the length of one of the semiaxes is equal to the sum of the other two.

Besides this, Morais et al. in [37] computed the coefficient of quasiconformality of those mappings. From the point of view taken here, this is particularly rewarding since the computation of this coefficient gives us the information of the ratio of the major to minor axes of the aforementioned ellipsoids. Further, dilatations and distortions of these mappings were estimated in [15]. In [17] it is proved that solid angles are not invariant under M-conformal mappings. It is shown that the change of the solid angle depends on one real parameter only. In particular, it is proved that M-conformal mappings orthogonal to all paramonogenic constants admit a certain change of solid angles and vice versa; that change can characterize such mappings. In spite of the fact that, M-conformal mappings are not conformal in the Gauss sense (i.e they do not preserve angles between curves, in general) we could show that there exist certain planes on $\mathbb{R}^{3}$ in which those mappings behave like conformal mappings in the complex plane, see [17].

We can say a little more. As pointed out in [16], there is a special geometric characterization of the subclass of monogenic mappings that are orthogonal to the (infinite dimensional subspace) of paramonogenic constants. Next we formulate the result.

Theorem 7 Let $f$ be a real analytic mapping from $\mathbb{R}^{3}$ to $\mathcal{A}_{2}$ with $\left.J_{f}(x)\right|_{x=0}$ $\neq 0$ and such that $\left.\left(1 / 2 \bar{D}_{2}\right) f(x)\right|_{x=0} \in \mathbb{R} \backslash\{0\}$. Then, $f$ is monogenic if and only if it locally maps small balls onto prolate spheroids with the property that the length of one of the semiaxes is the double of the other semiaxes.

It is well known that for holomorphic functions $f$, it is a classical result that the condition $f^{\prime}\left(z_{0}\right) \neq 0$ is both necessary and sufficient to ensure that $f$ realizes a local conformal mapping at the point $z_{0}$. Since in this case the Jacobian is $J_{f}(z)=\left|f^{\prime}(z)\right|^{2}$, it says that the Jacobian of $f$ cannot vanish at any point and does not depend on the direction of $f^{\prime}(z)$.

Remark 6 If $f: \mathbb{R}^{3} \rightarrow \mathcal{A}_{2}$ is monogenic and orthogonal to the paramonogenic constants then it holds:

$$
\left.J_{f}(x)\right|_{x=0} \neq\left. 0 \Longleftrightarrow\left(\frac{1}{2} \overline{D_{2}}\right) f(x)\right|_{x=0} \neq 0 .
$$

The last remark relates the characterization of M-conformal mappings by means of the Jacobian, as is usual in the general theory of quasiconformal mappings, with the hypercomplex derivative. These characterizations are equivalent in the mentioned subclass but not "identical" as in classical complex analysis. 


\subsection{The Nonsingular Case}

In the present subsection we extend the above results to the corresponding situation in $\mathbb{R}^{n+1}$ when $n>2$. More precisely, in Theorem 8 below we show that every $f \in \mathcal{M}_{n}$ with nonvanishing Jacobian determinant locally maps the unit sphere onto explicitly characterized ellipsoids and vice versa. To do so, we need the following technical lemma.

Lemma 1 Let $\Delta=\operatorname{diag}\left(\lambda_{1}, \ldots, \lambda_{n+1}\right)$. We assume that $\Delta$ is traceless, $\lambda_{1} \geq$ $\cdots \geq \lambda_{n+1}$ and that $\operatorname{det} \Delta<0$. We have the possibilities:

1. if $n=2 m$ so that $n+1=2 m+1$ then

$$
\lambda_{1}+\cdots+\lambda_{2 p}=-\left(\lambda_{2 p+1}+\cdots+\lambda_{2 m+1}\right),
$$

for $p=1, \ldots, m, m=1,2, \ldots$;

2. if $n=2 m+1$ so that $n+1=2 m+2$ then

$$
\lambda_{1}+\cdots+\lambda_{2 p-1}=-\left(\lambda_{2 p}+\cdots+\lambda_{2 m+2}\right),
$$

for $p=1, \ldots, m+1, m=0,1, \ldots$.

Similar results hold when $\operatorname{det} \Delta>0$.

Proof Newton's identities (Vieta's formulae) relate the eigenvalues of $\Delta$ in the following way: $\operatorname{det} \Delta=\lambda_{1} \cdots \lambda_{n+1}$, and $\operatorname{tr} \Delta=\lambda_{1}+\cdots+\lambda_{n+1}=0$. Hereby $\operatorname{tr} \Delta$ denotes the trace of $\Delta$. Since $\operatorname{det} \Delta<0$, we have an odd number of negative eigenvalues and with ordered eigenvalues we must then have $\lambda_{n+1}<0$. Since $\operatorname{tr} \Delta=0$, we must have at least one positive eigenvalue. We then get the conditions on the eigenvalues.

For convenience, in what follows, we will restrict ourselves to ellipsoids centered at the origin. In fact, this assumption involves no loss of generality if the center is known since, given a noncentered ellipsoid, we may always translate our coordinate system and therefore recover the centered case. We are thus led to the following result that generalizes the results in [16]:

Theorem 8 Suppose that $f \in \mathcal{M}_{n}$ with matrix $A$ and $\operatorname{det} A>0$. Then $f$ maps the unit sphere onto an ellipsoid. If we assume that the semiaxes are ordered as $A_{1} \geq A_{2} \geq \cdots \geq A_{n+1}>0$, then there is a permutation $\sigma \in \mathcal{S}_{n+1}$ so that with $B_{k}=\sigma\left(A_{k}\right)$ one of the following holds:

1. if $n=2 m$, so that $n+1=2 m+1$ we have the possibilities

$$
B_{1}+\cdots+B_{2 p}=B_{2 p+1}+\cdots+B_{2 m+1},
$$

for $p=1, \ldots, m, m=1,2, \ldots$;

2. if $n=2 m+1$, with $n+1=2 m+2$, then

$$
B_{1}+\cdots+B_{2 p-1}=B_{2 p}+\cdots+B_{2 m+2},
$$

for $p=1, \ldots, m+1, m=0,1, \ldots$. 
Moreover, given numbers $A_{1} \geq A_{2} \geq \cdots \geq A_{n+1}>0$ and a permutation $\sigma \in \mathcal{S}_{n+1}$ with $B_{k}=\sigma\left(A_{k}\right)$ such that $\left\{B_{k}\right\}$ satisfies one of the conditions in 1. or 2., there is a function $f \in \mathcal{M}_{n}$, such that $f$ maps the unit sphere onto an ellipsoid whose axes satisfy the condition. Similar results hold when $\operatorname{det} A<0$.

Proof Suppose that $D_{n} f=0$. Then $\tilde{A}=R_{n} A$ is symmetric and traceless. Hence $A=R_{n} \mathcal{O} \Delta \mathcal{O}^{T}$ where $\Delta$ is diagonal. Notice that $\operatorname{det} \Delta=\operatorname{det} \tilde{A}=$ $-\operatorname{det} A$. The absolute value of the eigenvalues are the lengths of the semiaxes of the image of the unit sphere. The converse results for example using diagonal matrices.

Remark 7 It is interesting to note that in the cases $n+1=4 m+2$ it is possible for there to be an equal number of positive and negative eigenvalues and it is then also possible for them to have equal absolute values. In this case $\Delta$ is a dilation with reflections.

Remark 8 This result allows us to describe M-conformal mappings as a special class of quasiconformal mappings. If we visualize quasiconformal mappings in $\mathbb{R}^{n+1}$ by points, given by the lengths of the semiaxes of the associated ellipsoids, then the monogenic functions (with nonvanishing Jacobian determinant) can be seen as an $n \mathrm{D}$ manifold in $\mathbb{R}^{n+1}$.

At this stage it seems natural to ask whether or not there exists another formulation of Theorem 8 for M-conformal mappings, depending on properties of the hypercomplex derivative. We begin with the preliminary result:

Lemma 2 Suppose that $f \in \mathcal{M}_{n}$. Then we have

$$
\left.J_{f}(x)\right|_{x=0} \neq\left. 0 \Longrightarrow\left(\frac{1}{2} \bar{D}_{n}\right) f(x)\right|_{x=0} \neq 0 .
$$

Proof We can follow directly the ideas from [7]. If $\left.\left(\frac{1}{2} \bar{D}_{n}\right) f(x)\right|_{x=0}=0$ then we get immediately from the monogenicity that $\frac{\partial f}{\partial x_{0}}=0$ and this implies $\left.J_{f}(x)\right|_{x=0}=0$.

We now estimate the distortions of an M-conformal mapping. For the estimation of the terms $\max _{|x|=r}|f(x)-f(0)|$ and $\min _{|x|=r}|f(x)-f(0)|$ it is enough to consider only $\left|\sum_{l=0}^{n} x_{l} \partial x_{l} f(x)\right|_{x=0} \mid$ for the maximum and minimum on the surface of the ball $|x|=r$. A direct computation shows that $\left(\lambda^{2}\right)_{\min } \leq\left|\left(\frac{1}{2} \bar{D}_{n}\right) f\right|^{2} \leq \operatorname{tr}\left(J_{f}^{T} J_{f}\right)=\sum_{i=1}^{n+1} \lambda_{i}^{2}$. We remark that because of the monogenicity $\sum_{i=1}^{n+1} \lambda_{i}=0$ holds and, furthermore, the eigenvalues are related to each other by Theorem 8 . On the other hand, using the Rayleigh-quotient it follows that

$$
\left(\lambda^{2}\right)_{\min }=\min _{|x| \neq 0} \frac{X^{T}\left(J_{f}^{T} J_{f}\right) X}{X^{T} X} \leq \frac{Y^{T}\left(J_{f}^{T} J_{f}\right) Y}{Y^{T} Y}, \quad Y \in \mathbb{R}^{n+1} .
$$

By choosing $Y=(1,0, \ldots, 0)^{T}$ we get finally

$$
\sqrt{\frac{\left(\lambda^{3}\right)_{\min }}{\prod_{i \neq \min }^{n+1} \lambda_{i}}} \sqrt{\left|J_{f}(0)\right|} \leq\left|\left(\frac{1}{2} \bar{D}_{n}\right) f\right|^{2} \leq \frac{\sum_{i=1}^{n+1} \lambda_{i}^{2}}{\sqrt{\prod_{i=1}^{n+1} \lambda_{i}}} \sqrt{\left|J_{f}(0)\right|}
$$


as well as

$$
\sqrt{\frac{\left(\lambda^{3}\right)_{\min }}{\prod_{i \neq \min }^{n+1} \lambda_{i}}}\left|\left(\frac{1}{2} \bar{D}_{n}\right) f\right|^{2} \leq \sqrt{\left|J_{f}(0)\right|} \leq \frac{\sum_{i=1}^{n+1} \lambda_{i}^{2}}{\sqrt{\prod_{i=1}^{n+1} \lambda_{i}}}\left|\left(\frac{1}{2} \bar{D}_{n}\right) f\right|^{2} .
$$

These inequalities show the equivalence of the Jacobian determinant and the hypercomplex derivative of a monogenic function.

Using the above results we summarize.

Theorem 9 Let $A$ be an $(n+1) \times(n+1)$ nonsingular matrix with $\operatorname{det} A>0$ so that $R_{n} A$ is symmetric and traceless and define $f(x)=A x$. Then $f \in \mathcal{M}_{n}$ and $f$ is an $M$-conformal mapping of $\mathbb{R}^{n+1}$ to itself. If further $A$ is orthogonal, then $R_{n} A$ is also orthogonal, and since it is also symmetric, $f$ is a reflection and so is conformal. Notice in this case $f$ maps the unit ball to itself. Similar results hold when $\operatorname{det} A<0$.

Theorem 9 is not only true for linear mappings, but hold for general monogenic mappings as well because their local behaviors are completely determined by their linear parts. From the point of view taken here, this result provides a novel and promising way to a deeper understanding of M-conformal mappings as tools for quasiconformal mappings in higher dimensions. Just as importantly, it can be used later to describe the global behavior of these mappings.

\subsection{Orthogonal Spaces}

In our point of view there are several arguments to accept the class of monogenic $\mathcal{A}_{n}$-valued functions orthogonal to the subspace of paramonogenic mappings, $\mathcal{H}_{n}$, as very well-adapted to the class of holomorphic functions in the plane (for an account of such an argument, see [14,15] and [33, Chap. III]). The question arises as to whether there is a special geometric characterization of this subclass of M-conformal mappings. The answer is given in the following theorem.

Theorem 10 Suppose that $f \in \mathcal{M}_{n}$ so that $\left.\left(1 / 2 \bar{D}_{n}\right) f(x)\right|_{x=0} \in \mathbb{R} \backslash\{0\}$. Then $f$ is orthogonal to the subspace of paramonogenic mappings if and only if it assymptotically maps the unit sphere onto a prolate spheroid with the property that the length of one of the semiaxes is an $n$-multiple of the other semiaxes.

Proof The proof follows from Theorems 4 and 8 .

In the sequel, we describe the interplay between the Jacobian determinant and the hypercomplex derivative of this subclass of M-conformal mappings.

Theorem 11 Suppose that $f \in \mathcal{M}_{n}$. If $f$ is orthogonal to the subspace of paramonogenic mappings then we have

$$
\left.J_{f}(x)\right|_{x=0} \neq\left. 0 \Longleftrightarrow\left(\frac{1}{2} \overline{D_{n}}\right) f(x)\right|_{x=0} \neq 0 .
$$


Proof For the reverse we manage it by indirect proof. As in the complex case, we assume the initial condition $\left.\left(1 / 2 \bar{D}_{n}\right) f(x)\right|_{x=0} \in \mathbb{R} \backslash\{0\}$. From Theorem 4 we know that a general linear monogenic $\mathcal{A}_{n}$-valued function orthogonal to the subspace of paramonogenic mappings has the form $a\left(n x_{0}+\sum_{i=1}^{n} x_{i} e_{i}\right)$, with $a \in \mathbb{R}$. Hence it is clear that for the first step in the approximation process $\left.J_{f}(x)\right|_{x=0}=0$ implies $\left|\left(\frac{1}{2} \bar{D}_{n}\right) f\right|^{2}=0$, hence $\left.J_{f}(x)\right|_{x=0}=0$ does not depend on the direction of $\left(\frac{1}{2} \bar{D}_{n}\right) f$ (compare for the $\mathbb{R}^{4}$ case with [7]).

\section{Radial Mappings and the Cauchy Kernel}

This section discusses quasiconformal radial mappings and their relations with the Cauchy kernel and $p$-monogenic mappings.

The radial mappings, for $\alpha \neq 1$, given by

$$
\rho(x)=\frac{x}{|x|^{\alpha}},
$$

where $|x|^{2}=x_{0}^{2}+x_{1}^{2}+\cdots+x_{n}^{2}$, are diffeomorphisms from $\mathbb{R}^{n+1} \backslash\{0\}$ to itself. It is the conformal inversion when $\alpha=2$. Otherwise it is quasiconformal. It shall be noted that this map can be realized as a $p$-monogenic mapping (see [21]), namely if

$$
\mathcal{C}_{\alpha}(x)=\overline{\Phi(\rho(x))}=\frac{\bar{x}}{|x|^{\alpha}},
$$

then we have $D_{n}\left|\mathcal{C}_{\alpha}(x)\right|^{(p-2)} \mathcal{C}_{\alpha}(x)=0$, where $p=\frac{(\alpha+n-1)}{(\alpha-1)}$. When $\alpha=n+$ 1 , so that $p=2$, then the conjugated radial map is the classical Cauchy kernel and is monogenic outside the origin. If $\alpha=2$, then $p=n+1$ and the reflected conformal inversion satisfies the $(n+1)$ - Cauchy-Riemann equation; when $0<\alpha<1, \mathcal{C}_{\alpha}(x)$ is a $p$-monogenic quasiconformal mapping of the unit ball $B^{(n+1)}$ onto itself. The dilatations of $\mathcal{C}_{\alpha}(x)$ can be explicitly calculated from its eigenvalues. Notice that these dilations depend only on $\alpha$ and $n$. The eigenvectors determine the directions of the distortions.

Theorem 12 The dilatation of $\mathcal{C}_{\alpha}(x)$ is $n^{n}$ at each point of $\mathbb{R}^{n+1} \backslash\{0\}$. Hence $\mathcal{C}_{\alpha}(x)$ is $n^{n}$-quasiconformal. Moreover, the distortion is in the radial direction.

Proof It is clear that $\mathcal{C}_{\alpha}(x)$ is injective. A straightforward calculation shows that for $|y|=1$ and $c>0$,

$$
R_{n} \mathcal{C}_{\alpha}^{\prime}(c y)=\left(y, u_{1}, \ldots u_{n}\right) M\left(y, u_{1}, \ldots, u_{n}\right)^{T},
$$

where

$$
M:=\left(\begin{array}{cccc}
(\alpha-1) c^{-\alpha} & 0 & \cdots & 0 \\
0 & -c^{\alpha} & \cdots & 0 \\
\vdots & \vdots & \ddots & \vdots \\
0 & 0 & \ldots & -c^{\alpha}
\end{array}\right)
$$

and $\left\{u_{1}, \ldots, u_{n}\right\}$ is any orthonormal basis of the $-c^{\alpha}$ eigenspace. 
Next we describe the $3 \mathrm{D}$-case in some detail. For $x \in \mathbb{R}^{3}$, a direct computation shows that

$$
\mathcal{C}_{\alpha}^{\prime}(x)=\frac{1}{r^{(\alpha+2)}}\left(\begin{array}{ccc}
r^{2}-\alpha x_{0}^{2} & -\alpha x_{0} x_{1} & -\alpha x_{0} x_{2} \\
\alpha x_{1} x_{0} & -r^{2}+\alpha x_{1}^{2} & \alpha x_{1} x_{2} \\
\alpha x_{2} x_{0} & \alpha x_{2} x_{1} & -r^{2}+\alpha x_{2}^{2}
\end{array}\right),
$$

and so

$$
\begin{aligned}
& \mathcal{C}_{\alpha}^{\prime}(1,0,0)=R_{2}\left(\begin{array}{ccc}
\alpha-1 & 0 & 0 \\
0 & -1 & 0 \\
0 & 0 & -1
\end{array}\right), \\
& \mathcal{C}_{\alpha}^{\prime}\left(\frac{1}{\sqrt{3}}, \frac{1}{\sqrt{3}}, \frac{1}{\sqrt{3}}\right)=R_{2}\left(\begin{array}{ccc}
\frac{\alpha}{3}-1 & \frac{\alpha}{3} & \frac{\alpha}{3} \\
\frac{\alpha}{3} & \frac{\alpha}{3} \frac{-}{3} & \frac{\alpha}{3} \\
\frac{\alpha}{3} & \frac{\alpha}{3} & \frac{\alpha}{3}-1
\end{array}\right)
\end{aligned}
$$

The eigenvalues are again $\alpha-1,-1,-1$. It is notable that the distortion is in the radial direction. A special case for Dirac analysis is the Cauchy kernel when $\alpha=n+1$.

For example when $\alpha=3$, we find

Example 2 For $x \in \mathbb{R}^{3}$, let

$$
\mathcal{C}_{3}^{\prime}(x)=\left(\begin{array}{ccc}
\frac{1}{|x|^{5}} & 0 & 0 \\
0 & \frac{1}{|x|^{5}} & 0 \\
0 & 0 & \frac{1}{|x|^{5}}
\end{array}\right)\left(\begin{array}{ccc}
|x|^{2}-3 x_{0}^{2} & -3 x_{0} x_{1} & -3 x_{0} x_{2} \\
3 x_{1} x_{0} & -|x|^{2}+3 x_{1}^{2} & 3 x_{1} x_{2} \\
3 x_{2} x_{0} & 3 x_{2} x_{1} & -|x|^{2}+3 x_{2}^{2}
\end{array}\right) .
$$

Moreover, the Jacobian is given by $J_{\mathcal{C}_{3}}(x)=-\frac{2}{|x|^{9}}$. For example, we find

$$
\mathcal{C}_{3}^{\prime}(1,0,0)=R_{2}\left(\begin{array}{ccc}
2 & 0 & 0 \\
0 & -1 & 0 \\
0 & 0 & -1
\end{array}\right)
$$

and

$$
\begin{aligned}
\mathcal{C}_{3}^{\prime}\left(\frac{1}{\sqrt{3}}, \frac{1}{\sqrt{3}}, \frac{1}{\sqrt{3}}\right)=\left(\begin{array}{ccc}
0 & -1 & -1 \\
1 & 0 & 1 \\
1 & 1 & 0
\end{array}\right) & =R_{2}\left(\begin{array}{lll}
0 & 1 & 1 \\
1 & 0 & 1 \\
1 & 1 & 0
\end{array}\right) \\
& =R_{2} \mathcal{O}\left(\begin{array}{ccc}
2 & 0 & 0 \\
0 & -1 & 0 \\
0 & 0 & -1
\end{array}\right) \mathcal{O}^{T}
\end{aligned}
$$

where

$$
\mathcal{O}=\left(\begin{array}{lll}
\frac{1}{\sqrt{3}} & \frac{1}{\sqrt{2}} & \frac{\sqrt{2}}{2 \sqrt{3}} \\
\frac{1}{\sqrt{3}} & -\frac{1}{\sqrt{2}} & \frac{\sqrt{2}}{2 \sqrt{3}} \\
\frac{1}{\sqrt{3}} & 0 & -\frac{\sqrt{2}}{\sqrt{3}}
\end{array}\right)
$$

Hence the Cauchy kernel in $\mathbb{R}^{3}$ has dilatation 4 at each point in $\mathbb{R}^{3} \backslash\{0\}$. 


\section{Numerical Examples}

In this section we present a few numerical examples, produced by Maple, for computing the image of spheres of a given radius under a special subclass of nonlinear M-conformal mappings.

It would seem natural to begin the investigation of mappings built up from two simple types of functions such as were discussed in Sections 3 and 4 . We shall take up the sum of a linear monogenic mapping and the classical Cauchy kernel, whose study will require us to master a new situation. We define the new subclass of nonlinear M-conformal mappings in $\mathbb{R}^{3}$ as follows:

Definition 10 Let $P$ be a monogenic linear mapping whose Jacobian is given by $(2)$, and $\mathcal{C}_{3}(x)$, the Cauchy kernel. Then

$$
w: \Omega \subset \mathbb{R}^{3} \backslash\{0\} \rightarrow \mathcal{A}_{2}, \quad w(x):=P(x)+\mathcal{C}_{3}(x),
$$

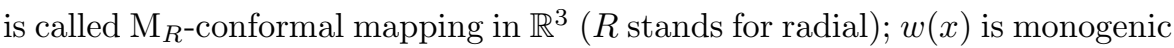
outside the origin.

Let $x \in \mathbb{R}^{3} \backslash\{0\}$. A direct computation shows that

$$
w^{\prime}(x)=\left(\begin{array}{ccc}
b_{1}+c_{2}+\frac{|x|^{2}-3 x_{0}^{2}}{|x|^{5}} & a_{1}-3 x_{0} x_{1} & a_{2}-3 x_{0} x_{2} \\
-a_{1}+3 x_{1} x_{0} & b_{1}-\frac{|x|^{2}-3 x_{1}^{2}}{|x|^{5}} & b_{2}+3 x_{1} x_{2} \\
-a_{2}+3 x_{2} x_{0} & b_{2}+3 x_{2} x_{1} & c_{2}-\frac{|x|^{2}-3 x_{2}^{2}}{|x|^{5}}
\end{array}\right),
$$

for $a_{i}, b_{i}, c_{2} \in \mathbb{R}(i=1,2)$. To facilitate our description, three distinct cases now present themselves:

(i) if $P$ is a paramonogenic mapping then $b_{1}+c_{2}=0$, and $a_{i}=0(i=1,2)$.

A direct computation shows that

$$
w^{\prime}(1,0,0)=R_{2} \mathcal{O}\left(\begin{array}{ccc}
-2 & 0 & 0 \\
0 & -1+\sqrt{b_{1}^{2}+b_{2}^{2}} & 0 \\
0 & 0 & -1-\sqrt{b_{1}^{2}+b_{2}^{2}}
\end{array}\right) \mathcal{O}^{T} .
$$

where $\mathcal{O}$ denotes the orthogonal matrix of eigenvectors. The eigenvalues are -2 , and $-1 \pm \sqrt{b_{1}^{2}+b_{2}^{2}}$. It is notable that the distortion is in the radial direction;

(ii) if $P$ is orthogonal to the subspace of paramonogenic mappings then $b_{1}=c_{2}$, and $b_{2}=0$. It follows that

$$
w^{\prime}(1,0,0)
$$

$$
=R_{2} \mathcal{O}\left(\begin{array}{ccc}
b_{1}-1 & 0 & 0 \\
0 & \frac{3\left(b_{1}-1\right)}{2}+\sqrt{\left(b_{1}-1\right)^{2}+4\left(a_{1}^{2}-a_{2}^{2}\right)} & 0 \\
0 & 0 & \frac{3\left(b_{1}-1\right)}{2}-\sqrt{\left(b_{1}-1\right)^{2}+4\left(a_{1}^{2}-a_{2}^{2}\right)}
\end{array}\right) \mathcal{O}^{T} ;
$$


(iii) if $P$ is orthogonal to the subspace of paramonogenic mappings and such that $\left.\left(1 / 2 \bar{D}_{2}\right) P(x)\right|_{x=0} \in \mathbb{R} \backslash\{0\}$ then

$$
w\left(x_{0}, x_{1}, x_{2}\right)=\left(2 b_{1}+\frac{1}{|x|^{3}}\right) x_{0}+\left(b_{1}-\frac{1}{|x|^{3}}\right)\left(x_{1} e_{1}+x_{2} e_{2}\right),
$$

with $b_{1} \in \mathbb{R}$. In this case we have

$$
w^{\prime}(1,0,0)=R_{2}\left(\begin{array}{ccc}
2 b_{1}-2 & 0 & 0 \\
0 & b_{1}-1 & 0 \\
0 & 0 & b_{1}-1
\end{array}\right) .
$$

We shall find it useful to introduce spherical coordinates $(\rho, \theta, \varphi)$, by setting $x_{0}=\rho \sin \varphi, x_{1}=\rho \cos \varphi \cos \theta$, and $x_{2}=\rho \cos \varphi \sin \theta$, with $\rho=|x|>0$, the radius of the sphere, $\theta$ the longitude so that $-\pi \leq \theta \leq \pi$, and $\varphi$ the latitude (not, like usually, the polar angle $\alpha=\frac{\pi}{2}-\varphi$ or co-latitude), $-\pi / 2 \leq \varphi \leq \pi / 2$. Let $w=w_{0}+w_{1} e_{1}+w_{2} e_{2}$; the mapping inferred from (3) by implementing the above coordinates, can be expressed by

$$
\begin{aligned}
& w_{0}=\left[\left(b_{1}+c_{2}\right) \rho+\frac{1}{\rho^{2}}\right] \sin \varphi+a_{1} \rho \cos \varphi \cos \theta+a_{2} \rho \cos \varphi \sin \theta, \\
& w_{1}=-a_{1} \rho \sin \varphi+\left(b_{1} \rho-\frac{1}{\rho^{2}}\right) \cos \varphi \cos \theta+b_{2} \rho \cos \varphi \sin \theta \\
& w_{2}=-a_{2} \rho \sin \varphi+b_{2} \rho \cos \varphi \cos \theta+\left(c_{2} \rho-\frac{1}{\rho^{2}}\right) \cos \varphi \sin \theta .
\end{aligned}
$$

This will give us a geometric picture of the mapping. From the geometrical and practical point of view, we are interested to map spheres to a domain in $\mathbb{R}^{3}$ (not necessarily a sphere). We can directly see that, in general, spheres are transformed onto ellipsoids. With a suitable choice of parameters and depending on the value of $\rho, 0<\rho<\infty$, we obtain oblate spheroids or prolate spheroids and for special limit cases even a sphere and the twofold-mapped $S^{1}$, including its interior, in the hyperplane $w_{0}=0$. We shall not carry through the details of such mappings, as this would lead us too far afield here. We shall, however, give a few examples that give us an insight into their geometric properties. Further investigations on this topic are now under investigation and will be reported in a forthcoming paper.

Remark 9 One fact that should be stressed here is that for a suitable choice of inputs to the Jacobian (2) the function (3) coincides (up to a real constant) with the 3D Joukowski monogenic transformation introduced in [31] (see Property (iii) above and Example 5 below).

We use this insight to motivate our numerical procedures for computing the image of spheres under $w(x)$. Let us take up a first example. 
Example 3 Take a mapping $w^{(1)}: \Omega \subset \mathbb{R}^{3} \backslash\{0\} \rightarrow \mathcal{A}_{2}$, which satisfies Property (i) above:

$$
\begin{aligned}
w^{(1)}(\rho, \theta, \varphi)=\frac{\sin \varphi}{\rho^{2}} & +\left[\left(\rho-\frac{1}{\rho^{2}}\right) \cos \varphi \cos \theta+\rho \cos \varphi \sin \theta\right] e_{1} \\
+ & {\left[\rho \cos \varphi \cos \theta-\left(\rho+\frac{1}{\rho^{2}}\right) \cos \varphi \sin \theta\right] e_{2} . }
\end{aligned}
$$

The next three figures visualize the image of a sphere whose radii are $1, \frac{1}{2}$, and $\sqrt[3]{4}$ under the mapping function $w^{(1)}$. Notice that a sphere of radius $\rho=1$ is mapped onto an oblate spheroid; a sphere of radius $\rho=\frac{1}{2}$ is mapped onto an ellipsoid, and a sphere of radius $\rho=\sqrt[3]{4}$ is mapped onto a tri-axial ellipsoid.
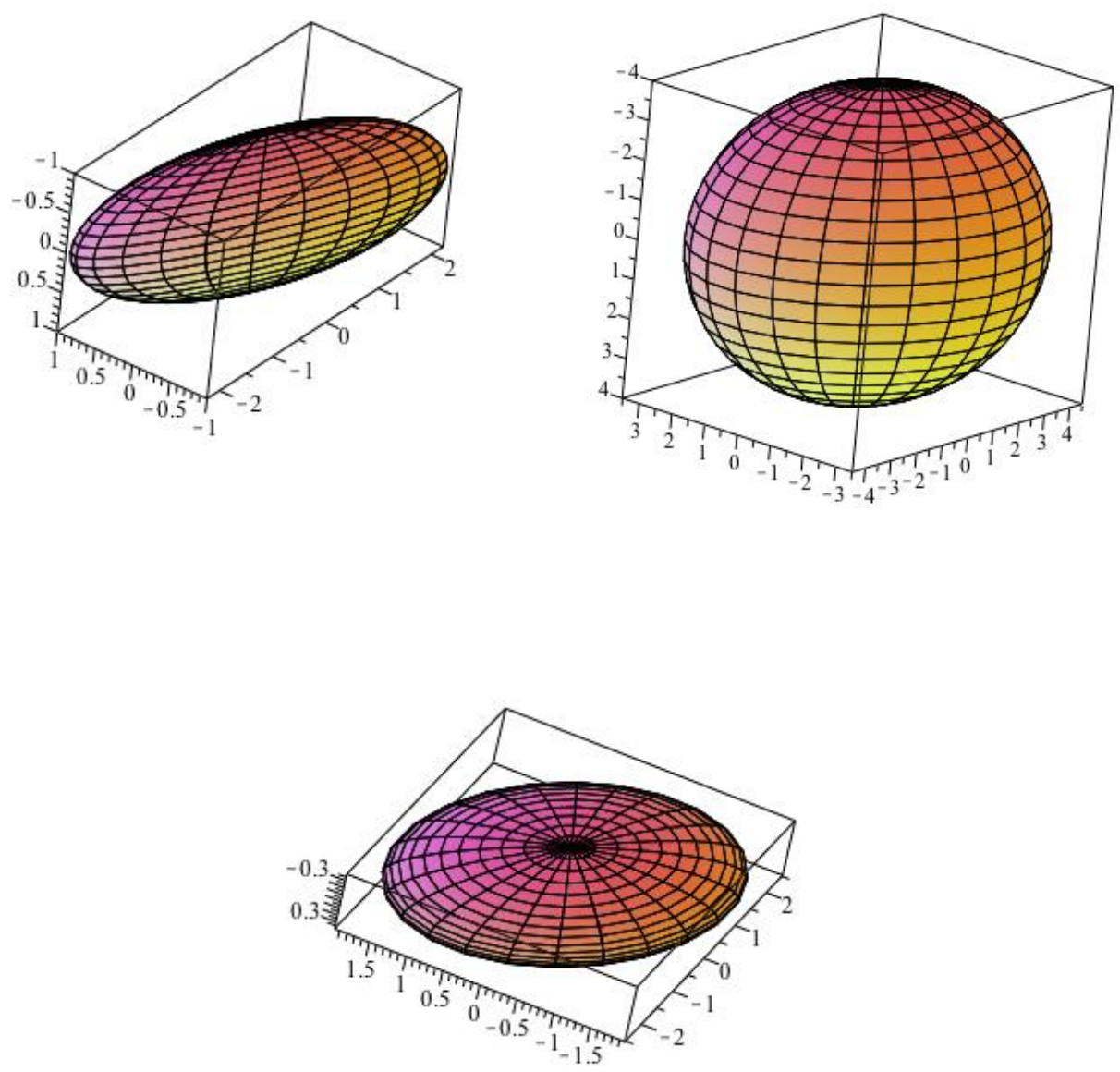
Example 4 Take a mapping $w^{(2)}: \Omega \subset \mathbb{R}^{3} \backslash\{0\} \rightarrow \mathcal{A}_{2}$, which satisfies Property (ii) above:

$$
\begin{aligned}
w^{(2)}(\rho, \theta, \varphi) & =\left(-2 \rho+\frac{1}{\rho^{2}}\right) \sin \varphi+\rho \cos \varphi \cos \theta-\left(\rho+\frac{1}{\rho^{2}}\right) \cos \varphi \sin \theta e_{2} \\
& -\left[\rho \sin \varphi+\left(\rho+\frac{1}{\rho^{2}}\right) \cos \varphi \cos \theta\right] e_{1}
\end{aligned}
$$

The next three figures visualize the image of a sphere whose radii are $1, \frac{1}{2}$, and $\sqrt[3]{4}$ under $w^{(2)}$. All spheres are mapped onto an ellipsoid.
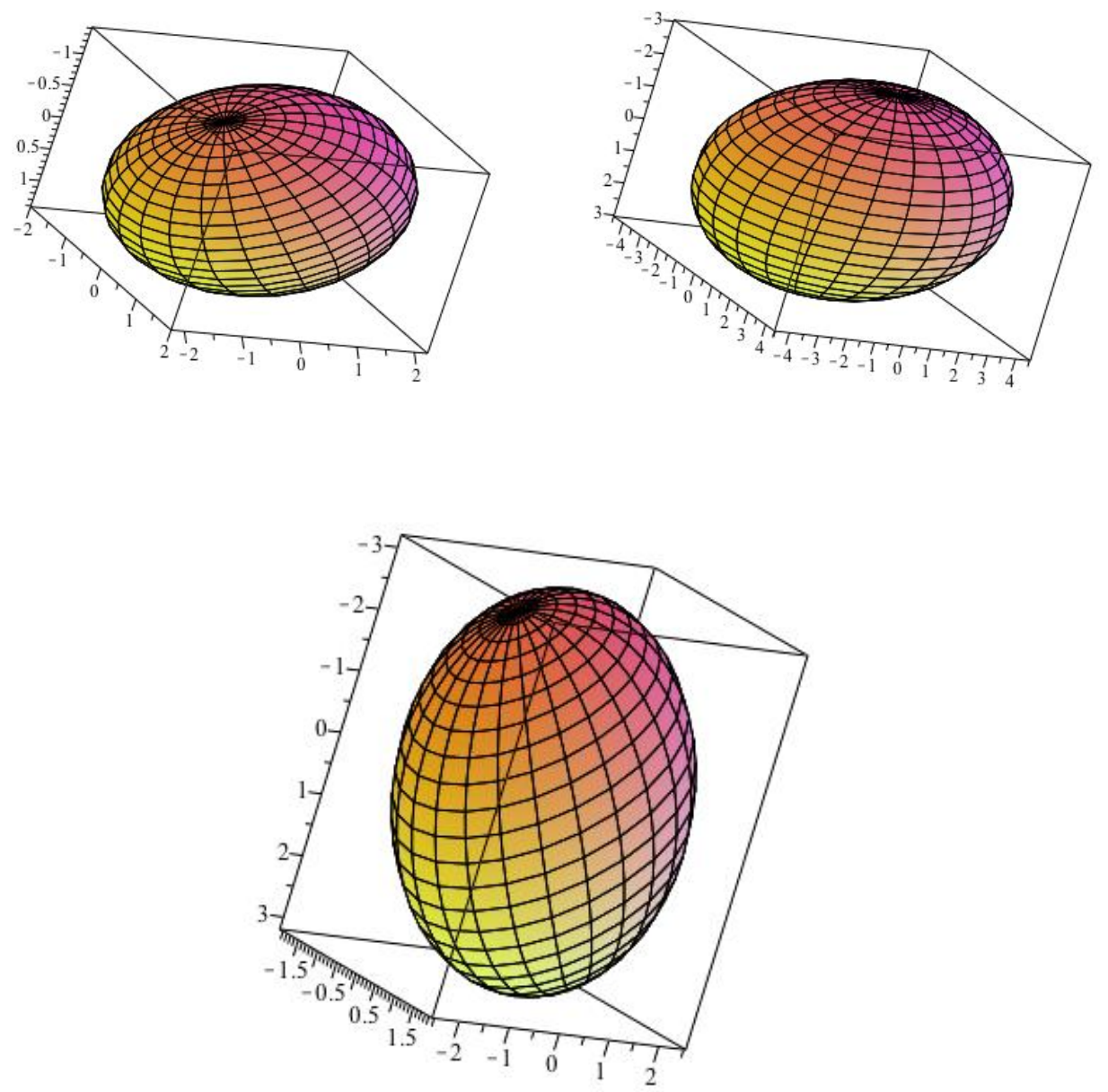
We now consider a mapping $w^{(3)}: \Omega \subset \mathbb{R}^{3} \backslash\{0\} \rightarrow \mathcal{A}_{2}$, which satisfies Property (iii) above. Notice that this function coincides (up to a real constant) exactly with the aforementioned Joukowski transformation. For more details the interested reader is suggested to check some of the existing pioneering works, see $[3,31]$.

Example 5 Take

$$
w^{(3)}(\rho, \theta, \varphi)=\left[\rho-\frac{1}{\rho^{2}}\right] \sin \varphi+\left(\frac{\rho}{2}+\frac{1}{\rho^{2}}\right) \cos \varphi\left(\cos \theta e_{1}+\sin \theta e_{2}\right) .
$$

The discussion of this function offers no particular difficulty. As the next three figures illustrate, the unit sphere $\rho=1$ is mapped into $\cos \varphi\left(\cos \theta e_{1}+\sin \theta e_{2}\right)$, i.e. the twofold-mapped $S^{1}$, including its interior, in the hyperplane $w_{0}=0$; a sphere of radius $\rho=\frac{1}{2}$ is mapped onto an oblate spheroid, and a sphere of radius $\rho=\sqrt[3]{4}$ is mapped onto a sphere of radius $\frac{4}{3}$.
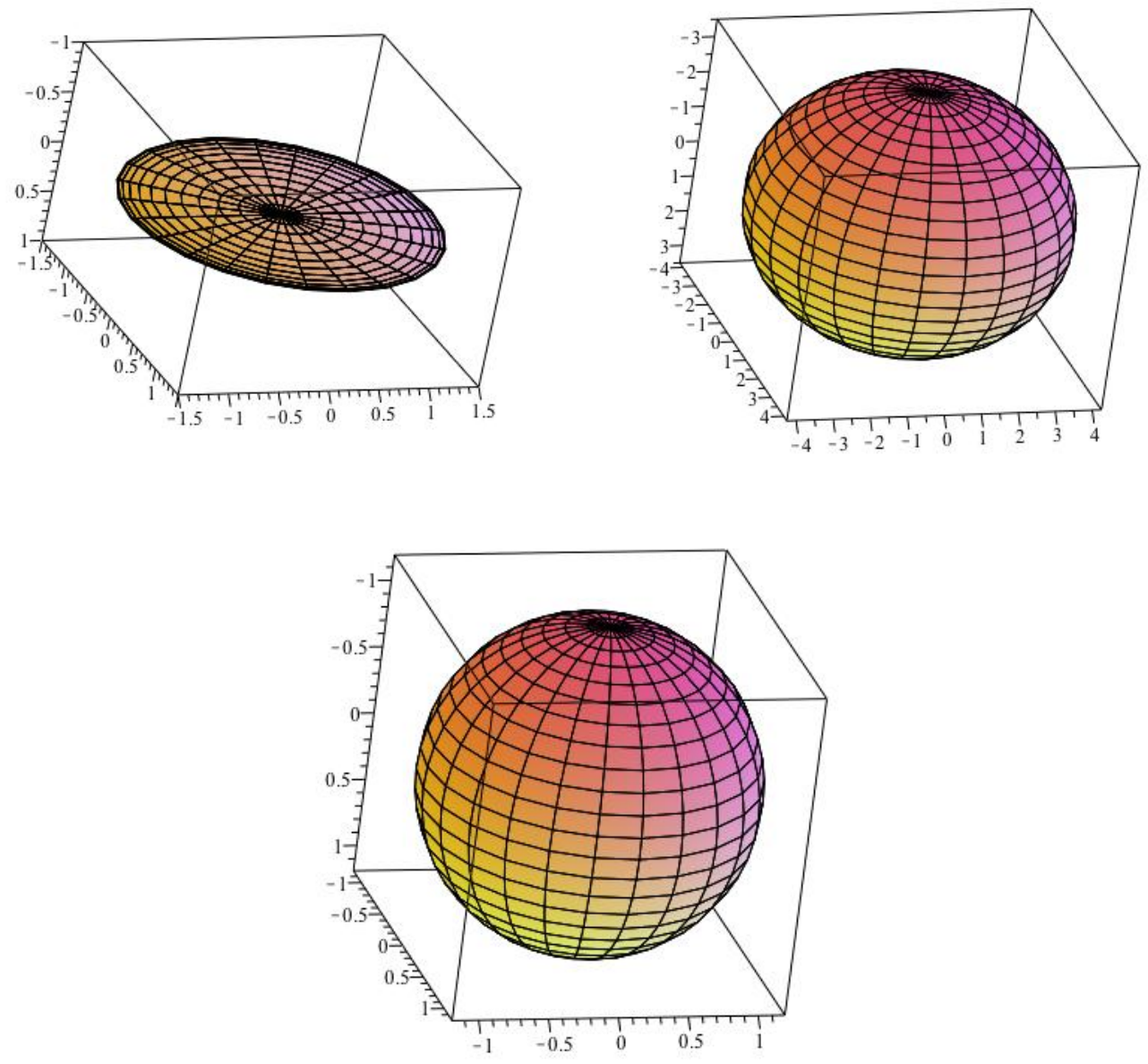


\section{Quadratic M-Conformal Mappings}

This section discusses the concept of quadratic M-conformal mapping and presents some numerical examples showing the image of the unit sphere under special quadratic monogenic functions.

We begin with the following definition.

Definition 11 A mapping $g: \mathbb{R}^{n+1} \rightarrow \mathcal{A}_{n}$ is quadratic M-conformal when it is of the form

$$
g(x):=\left(x A_{0} x^{t}, \ldots, x A_{n} x^{t}\right)
$$

where $A_{i}(i=0, \ldots, n)$ are symmetric $(n+1) \times(n+1)$ real matrices. The Jacobi matrix is then $J_{g}(x)=2\left(x A_{0}, x A_{1}, \ldots, x A_{n}\right)^{T}$.

Theorem 13 (see [41]) A quadratic mapping is a harmonic mapping if and only if each matrix $A_{i}(i=0, \ldots, n)$ is traceless.

Remark 10 It is easy to see that for a quadratic M-conformal mapping in a planar domain, the symmetric traceless matrices $A_{0}$ and $A_{1}$ also satisfy the relations $A_{0} A_{1}=-A_{1} A_{0}$, and $A_{0}^{2}=A_{1}^{2}=-c I$, where $c=\operatorname{det} A_{0}=\operatorname{det} A_{1}$.

We formulate the main result of this section.

Lemma 3 The mapping $g: \mathbb{R}^{n+1} \rightarrow \mathcal{A}_{n}$ given by (4) is quadratic $M$-conformal if and only if $R_{n} g^{\prime}(x)=2\left(-x A_{0}, x A_{1}, \ldots, x A_{n}\right)^{T}$ is symmetric and traceless for all $x \in \mathbb{R}^{(n+1)}$. Moreover $R_{n} g^{\prime}\left(u_{i}\right)=-2 A_{i}$.

Proof The proof follows from previous results.

Now consider the following examples that illustrate two cases of 3D quadratic M-conformal mappings.

Example 6 The quadratic monogenic function

$$
g(x)=\left(-2 x_{0}^{2}+x_{1}^{2}+x_{2}^{2}\right)-2 x_{0} x_{1} e_{1}-2 x_{0} x_{2} e_{2}
$$

is given by the matrices

$$
A_{0}=\left(\begin{array}{ccc}
-2 & 0 & 0 \\
0 & 1 & 0 \\
0 & 0 & 1
\end{array}\right), \quad A_{1}=\left(\begin{array}{ccc}
0 & -1 & 0 \\
-1 & 0 & 0 \\
0 & 0 & 0
\end{array}\right), \quad A_{2}=\left(\begin{array}{ccc}
0 & 0 & -1 \\
0 & 0 & 0 \\
-1 & 0 & 0
\end{array}\right) \text {. }
$$

In particular

$$
\begin{aligned}
& R_{2} g^{\prime}(1,0,0)=\left(\begin{array}{ccc}
4 & 0 & 0 \\
0 & -2 & 0 \\
0 & 0 & -2
\end{array}\right)=-2 A_{0}, \quad R_{2} g^{\prime}(1,1,0)=\left(\begin{array}{ccc}
4 & -2 & 0 \\
-2 & -2 & 0 \\
0 & 0 & -2
\end{array}\right), \\
& R_{2} g^{\prime}(1,2,3)=\left(\begin{array}{ccc}
4 & -4 & -3 \\
-4 & -2 & 0 \\
-3 & 0 & -2
\end{array}\right) .
\end{aligned}
$$


Example 7 The quadratic monogenic function

$h(x)=\left(2 x_{0} x_{1}+2 x_{0} x_{2}+2 x_{1} x_{2}\right)-\left(x_{0}^{2}+2 x_{0} x_{2}-x_{1}^{2}\right) e_{1}-\left(x_{0}^{2}+2 x_{0} x_{1}-x_{2}^{2}\right) e_{2}$

is determined by

$$
A_{0}=\left(\begin{array}{lll}
0 & 1 & 1 \\
1 & 0 & 1 \\
1 & 1 & 0
\end{array}\right), \quad A_{1}=\left(\begin{array}{ccc}
-1 & 0 & -1 \\
0 & 1 & 0 \\
-1 & 0 & 0
\end{array}\right), \quad A_{2}=\left(\begin{array}{ccc}
-1 & -1 & 0 \\
-1 & 0 & 0 \\
0 & 0 & 1
\end{array}\right)
$$

The next figures visualize the image of the unit sphere centered at the origin under the mappings $g$ and $h$, and lead to qualitatively very good numerical results.
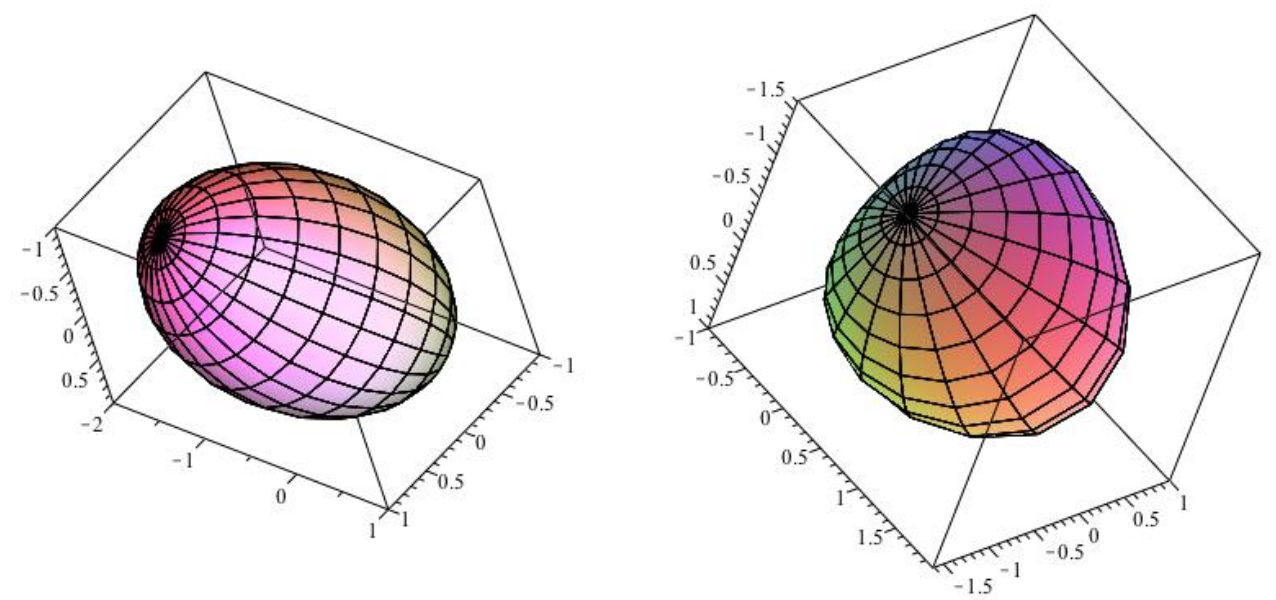

We are thus led to the following conjecture:

Conjecture 1 If $g: \mathbb{R}^{n+1} \rightarrow \mathcal{A}_{n}$ is a quadratic M-conformal mapping with symmetric traceless matrices $\left(A_{0}, \ldots, A_{n}\right)$, then the row matrix $R_{n}\left(A_{0}, \ldots, A_{n}\right)$ is symmetric and traceless.

\section{Split Complex Case}

We show in this section how the techniques above extend to other Clifford algebras.

Let $f: \Omega \subset \mathbb{R}^{2} \rightarrow \mathcal{C} \ell_{1,0}$ and $z=x+j y$. To begin with, we note that if $\bar{\nabla} f=0$ then

$$
f^{\prime}(z)=B=\left(\begin{array}{ll}
a & b \\
b & a
\end{array}\right)
$$


where $a=u_{x}$ and $b=u_{y}$. Also, $J_{f}=a^{2}-b^{2}$ is the Jacobian. We have the diagonalization

$$
\left(\begin{array}{ll}
a & b \\
b & a
\end{array}\right)=\left(\begin{array}{cc}
\sqrt{2} & \sqrt{2} \\
\sqrt{2} & -\sqrt{2}
\end{array}\right)\left(\begin{array}{cc}
a+b & 0 \\
0 & a-b
\end{array}\right)\left(\begin{array}{cc}
\sqrt{2} & \sqrt{2} \\
\sqrt{2} & -\sqrt{2}
\end{array}\right) .
$$

It is expected that the distortion is in the $x= \pm y$ directions as the calculations below show. Also when $a \neq \pm b$ the distortion is

$$
\max \left(\left|\frac{a+b}{a-b}\right|,\left|\frac{a-b}{a+b}\right|\right) .
$$

On account of Remark 1 we have the decomposition

$$
z=x+j y=(x+y) j^{+}+(x-y) j^{-}=X j^{+}+Y j^{-} .
$$

Also $z^{n}=X^{n} j^{+}+Y^{n} j^{-}$. We define $N(z)=x^{2}-y^{2}$.

Consider the matrix groups

$$
G L_{2}\left(\mathcal{C} \ell_{1,0}\right)=\left\{A=\left(\begin{array}{ll}
a & b \\
c & d
\end{array}\right) \mid a, b, c, d \in \mathcal{C} \ell_{1,0}, N(\operatorname{det} A) \neq 0\right\} .
$$

Writing

$$
A=\left(\begin{array}{ll}
a_{1} & b_{1} \\
c_{1} & d_{1}
\end{array}\right) j^{+}+\left(\begin{array}{ll}
a_{2} & b_{2} \\
c_{2} & d_{2}
\end{array}\right) j^{-}=A_{1} j^{+}+A_{2} j^{-},
$$

we have $\operatorname{det} A=\operatorname{det} A_{1} j^{+}+\operatorname{det} A_{2} j^{-}$, and $N(\operatorname{det} A)=\operatorname{det} A_{1} \operatorname{det} A_{2}$. It follows that

$$
G L_{2}\left(\mathcal{C} \ell_{1,0}\right)=G L_{2}(\mathbb{R}) \times G L_{2}(\mathbb{R}) .
$$

Moreover $\operatorname{det} A=1$ if and only if $\operatorname{det} A_{1}=\operatorname{det} A_{2}=1$, so that

$$
S L_{2}\left(\mathcal{C} \ell_{1,0}\right)=S L_{2}(\mathbb{R}) \times S L_{2}(\mathbb{R}) .
$$

We also have that $\operatorname{tr} A=\operatorname{tr} A_{1} j^{+}+\operatorname{tr} A_{2} j^{-}$. Hence the Möbius transformations act on the subspaces generated by $j^{ \pm}$.

With $X=x+y$, and $Y=x-y$, operators transform coordinates as

$$
\bar{\nabla}=j^{+} \partial_{Y}+j^{-} \partial_{X}, \quad \nabla=\partial_{X} j^{+}+\partial_{Y} j^{-} .
$$

Analytic functions also decompose

Theorem 14 Let $f=F_{1}+F_{2} j: \Omega \subset \mathbb{R}^{2} \rightarrow \mathcal{C} \ell_{1,0}$. The following are equivalent:

1. $\nabla f=0$;

2. $f(X, Y)=G_{1}(X) j^{+} G_{2}(Y) j^{-}$for real-valued functions $G_{1}, G_{2}$;

3. $\partial_{x} F_{1}=\partial_{u} F_{2}, \partial_{u} F_{1}=\partial_{x} F_{2}$. 
Recent studies have shown that our methods also allow a generalization of the distortion theory for Euclidean Clifford analytic functions, and for analytic functions in hyperbolic Clifford algebras. Besides their obvious importance these results will not be discussed in the present article. The authors are currently attempting to do this and at the same time exploring a class of periodic monogenic mappings. These period mappings are sums of the classical Cauchy kernel and its partial derivatives on $q \mathrm{D}$ lattices. There are analogues of periodic analytic functions to periodic monogenic functions on $q \mathrm{D}$ lattices in space, see [23].

\section{Acknowledgements}

The first author's work is supported by FEDER funds through COMPETEOperational Programme Factors of Competitiveness ("Programa Operacional Factores de Competitividade") and by Portuguese funds through the Center for Research and Development in Mathematics and Applications (University of Aveiro) and the Portuguese Foundation for Science and Technology ("FCT-Fundação para a Ciência e a Tecnologia"), within project PEstC/MAT/UI4106/2011 with COMPETE number FCOMP-01-0124-FEDER022690. Support from the Foundation for Science and Technology (FCT) via the post-doctoral grant SFRH/BPD/66342/2009 is also acknowledged by the first author.

\section{References}

1. L.V. Ahlfors. Zur Theorie der Überlagerungsflächen. Acta Math. 65, 157-194 (1935).

2. L.V. Ahlfors. Lectures on quasiconformal mappings. Van Nostrand, 1966.

3. R. Almeida and H. Malonek. On a Higher Dimensional Analogue of the Joukowski Transformation. AIP Conf. Proc. 1048, 630-633 (2008).

4. K. Astala, T. Iwaniec and G. Martin. Elliptic partial differential equations and quasiconformal mappings in the plane. Vol. 48 of Princeton Mathematical Series, Princeton University Press, Princeton, NJ, 2009.

5. S. Bock, M.I. Falcão, K. Gürlebeck and H. Malonek. A 3-Dimensional Bergman Kernel Method with Applications to Rectangular Domains. Journal of Computational and Applied Mathematics, Vol. 189, 6-79 (2006).

6. B. Boone. Bergmankern en conforme albeelding: een excursie in drie dimensies. Proefschrift, Universiteit Gent Faculteit van de Wetenshappen (1991-1992).

7. P. Cerejeiras, K. Gürlebeck, U. Käler and H. Malonek. A quaternionic Beltrami-type equation and the existence of local homeomorphic solutions. Journal for Analysis and Applcations, Vol. 20, No. 1, 17-34 (2001).

8. C. Cruz, M. I. Falcão, H. Malonek. 3D Mappings by Generalized Joukowski Transformations. Computational Science and Its Applications - ICCSA 2011, Lecture Notes in Computer Science, Volume 6784, 358-373, Santander (2011).

9. R. Delanghe, R. Kraußhar and H. Malonek. Differentiability of functions with values in some real associative algebras: approaches to an old problem. Bulletin de la Société Royale des Sciences de Liège, Vol. 70, No. 4-6, 231-249 (2001).

10. F.W. Gehring. Quasiconformal mappings in space. Bull. Amer. Math. Soc., Vol. 69, No. 2, 146-164 (1963).

11. J. Gilbert and M. Murray. Clifford Algebras and Dirac Operators in Harmonic Analysis. Cambridge University Press, 1991. 
12. H. Grötzsch. Über möglichst konforme Abbildungen yon schlichten Bereichen. Ber. Verh. Sächs. Akad. Wiss. Leipzig 84, 114-120 (1932).

13. K. Gürlebeck and H. Malonek. A hypercomplex derivative of monogenic functions in $\mathbb{R}^{n+1}$ and its applications. Complex Variables, Vol. 39, 199-228 (1999).

14. K. Gürlebeck and J. Morais. On mapping properties of monogenic functions. CUBO A Mathematical Journal, Vol. 11, No. 1, 73-100 (2009).

15. K. Gürlebeck and J. Morais. Local properties of monogenic mappings. AIP Conf. Proc., Numerical analysis and applied mathematics, Vol. 1168, 797-800 (2009).

16. K. Gürlebeck and J. Morais. Geometric characterization of M-conformal mappings. Geometric Algebra Computing: in Engineering and Computer Science, Bayro-Corrochano, Eduardo; Scheuermann, Gerik (Eds.), Springer, 1st Edition, 327-342 (2010).

17. K. Gürlebeck, M.H. Nguyen and J. Morais. On M-conformal mappings. AIP Conf. Proc. 1493, 674-677 (2012).

18. H. G. Haefeli. Hyperkomplexe Differentiale. Comment. Math. Helv. 20, 382-420 (1947).

19. T. Iwaniec and G. Martin. Goemetric Function Theory and Non-linear Analysis. Oxford University Press, 2001.

20. V. Kolehmainen, M. Lassas and P. Ola. Electrical impedance tomography problem with inaccurately known boundary and contact impedances. IEEE Transactions on Medical Imaging, Vol. 27, No. 10, 1404-1414 (2008).

21. V. Kolehmainen, M. Lassas and P. Ola. Calderón's inverse problem with an imperfectly known boundary and reconstruction up to a conformal deformation. SIAM J. Math. Anal., Vol. 42, No. 3, 1371-1381 (2010).

22. V. Kolehmainen, M. Lassas, P. Ola and S. Siltanen. Recovering boundary shape and conductivity in electrical impedance tomography. Inverse Problems and Imaging, Vol. 7, No. 1, 217-242 (2013).

23. R. Kraushar. Eisenstein Series in Clifford Analysis. Aachener Beiträge zur Matematik, Band 28, 2000.

24. R. Kraußhar and H. Malonek. A characterization of conformal mappings in $\mathbb{R}^{4}$ by a formal differentiability condition. Bulletin de la Société Royale des Sciences de Liège, Vol. 70, No. 1, 35-49 (2001)

25. M. A. Lavrent'ev. Sur une cIasse de representatations continues. Mat. Sb. 42, 407-427 (1935).

26. M. A. Lavrent'ev. Sur un critère differentiel des transformations homéomorphes des domains à trois dimensions. Dokl. Acad. Nank SSSR 20, 241-242 (1938).

27. O. Lehto and K. Virtanen. Quasiconformal Mappings in the Plane. Springer-Verlag, Berlin, 1971

28. M. E. Luna Elizarrarás and M. Shapiro. A survey on the (hyper)derivates in complex, quaternionic and Clifford analysis. Millan J. of Math., Vol. 79, No. 2, 521-542 (2011).

29. H. Malonek. Contributions to a geometric function theory in higher dimensions by Clifford analysis methods: Monogenic functions and M-conformal mappings. In: Clifford Analysis and its Applications ed. F. Brackx et al., Kluwer, NATO Sci. Ser. II, Math. Phys. Chem. 25, 213-222 (2001).

30. H. Malonek and M.I Falcão. 3D-mappings by means of monogenic functions and their approximation. Mathematical Methods in the Applied Sciences, Vol. 33, No. 4, 423-430 (2010).

31. H. Malonek and R. Almeida. A note on a generalized Joukowski transformation. Applied Mathematics Letters, Vol. 23, 1174-1178 (2010).

32. I. Mitelman and M. Shapiro. Differentiation of the Martinelli-Bochner integrals and the notion of hyperderivability. Math. Nachr., Vol. 172, No. 1, 211-238 (1995).

33. J. Morais. Approximation by homogeneous polynomial solutions of the Riesz system in $\mathbb{R}^{3}$. Ph.D. thesis, Bauhaus-Universitat Weimar, 2009.

34. J. Morais and K. Gürlebeck. Bloch's theorem in the context of quaternion analysis. Computational Methods and Function Theory, Vol. 12, No. 2, 541-558 (2012).

35. J. Morais and C. Nolder. Local distortion of monogenic functions. AIP Conf. Proc. 1493, 703-709 (2012).

36. J. Morais, K.I. Kou and W. Sprössig. Generalized holomorphic Szegö kernel in 3D spheroids. Computers and Mathematics with Applications, Vol. 65, No. 4, 576-588 (2013).

37. J. Morais and M. Ferreira. Quasiconformal mappings in 3D by means of monogenic functions. Mathematical Methods in the Applied Sciences. doi: 10.1002/mma.2625. 
38. C.A. Nolder and J. Ryan. p-Dirac equations. Advances in Applied Clifford Algebras, Vol. 19, No.2, 391-402 (2009).

39. C.A. Nolder. A-harmonic equations and the Dirac operator. Journal of Inequalities and Applications, Article ID 124018, 9 pages (2010).

40. C.A. Nolder. Nonlinear A-Dirac equations. Advances in Applied Clifford Algebras, Vol. 21, No. 2, 429-440 (2011).

41. Ye-Lin Ou. On the classification of quadratic harmonic morphisms between euclidean spaces. Algebras, Groups Geom., Vol. 13, 41-53 (1996).

42. S. Rickman. Quasiregular mappings. Ergebnisse der Mathematik und ihrer Grenzgebiete 26, Springer-Verlag, Berlin - Heidelberg - New York, 1993.

43. M. Riesz. Clifford numbers and spinors. Institute for Physical Science, Vol. 54, Kluwer Academic Publishers, Dorrecht, 1993.

44. E. M. Stein and G. Weiß. On the theory of harmonic functions of several variables. Part I: The theory of $H^{p}$ spaces. Acta Math. 103, 25-62 (1960).

45. E.M Stein and G. Weiss. Generalization of the Cauchy-Riemann equations and representations of the rotation group. Amer. J. Math. 90, 163-196 (1968).

46. A. Sudbery. Quaternionic analysis. Math. Proc. Cambridge Phil. Soc. 85, 199-225 (1979).

47. O. Teichmüller. Untersuchungen über konforme und quasikonforme Abbildung, Deutsche Math. 3, 621-678 (1938).

48. J. Väisälä. On quasiconformal mappings of a ball, Ann. Acad. Sci. Fenn. Ser. A I Math. 304, 1-17 (1961).

49. J. Väisälä. Lectures on n-dimensional quasiconformal mappings, Lecture Notes in Mathematics 229, Springer-Verlag, 1971. 